\title{
Cloud-basierte Plattformen zur Anwendungsintegration - Angebote und Praxisbeispiel
}

\author{
Nico Ebert · Kristin Weber
}

Eingegangen: 4. Juni 2015 / Angenommen: 26. September 2015 / Online publiziert: 12. Oktober 2015

(C) Springer Fachmedien Wiesbaden 2015

Zusammenfassung Der Einsatz von Software-as-a-Service in Unternehmen beschränkt sich nicht mehr nur auf einzelne Pilotprojekte, sondern betrifft zunehmend auch geschäftskritische Anwendungen. Damit entsteht gleichzeitig der Wunsch nach Integration von Software-as-a-Service, etwa der CRM-Lösung Salesforce.com, und traditionellen On-Premise-Anwendungen wie SAP ERP. Diesen Bedarf versprechen „Cloud-basierte Integrationsplattformen“ flexibel und kostengünstig zu adressieren - als Alternative zur direkten Anbindung der Anwendungen oder zu klassischen Enterprise Application Integration-Plattformen. Sie bieten zahlreiche Anwendungsadapter und erlauben den grafischen Entwurf, die Ausführung und die Verwaltung von komplexen Integrationsprozessen in der Cloud. Einige Hersteller liefern Software-Agenten für die sichere Kommunikation zwischen Software-as-a-Service, OnPremise-Anwendungen und Integrationsplattform. Bei manchen Herstellern sind diese Agenten auch die lokale Laufzeitumgebung für Integrationsprozesse, weswegen dann keine kritischen Daten an die Integrationsplattform übertragen werden müssen.

Schlüsselwörter Software-as-a-Service · Anwendungsintegration · Enterprise Application Integration · Cloud-basierte Integrationsplattform · Integration-Platform-as-a-Service

\footnotetext{
N. Ebert $(\bowtie)$

LearningCulture $\mathrm{GmbH}$,

Schubertstrasse 9,

8037 Zürich, Schweiz

E-Mail: nico.ebert@gmail.com

K. Weber

Fakultät Informatik und Wirtschaftsinformatik,

FHWS - Hochschule für angewandte Wissenschaften Würzburg-Schweinfurt,

Sanderheinrichsleitenweg 20,

97074 Würzburg, Deutschland
} 
Abstract The use of Software-as-a-Service in the company context is no longer limited to single pilot projects but increasingly includes business critical applications. This leads to a growing demand for the integration of Software-as-a-Service (e.g. Salesforce.com) and traditional on-premise applications. Cloud-based integration platforms promise to address this issue in flexible and cost-effective manner as an alternative to point-to-point integration or traditional enterprise application integration platforms. The cloud-based platforms offer a variety of standard application adapters and allow the visual development, the execution and the administration of complex integration processes within the cloud. Some vendors offer software agents for the secure communication between Software-as-a-Service, on-premise applications and the platforms. A few agents act as full local runtime environments for integration processes so that no critical data is shared with the platform.

\section{Bedarf für Cloud-basierte Integrationsplattformen}

Die Bedeutung von Software-as-a-Service (SaaS) für Unternehmen ist in den vergangen Jahren gestiegen. Der Einsatz von SaaS beschränkt sich nicht mehr nur auf ausgewählte Pilotprojekte, sondern umfasst zunehmend auch geschäftskritische Anwendungen im Unternehmen. Sogar der langfristige Ersatz bestehender ERP-Systeme durch SaaS wird in vielen Unternehmen bereits diskutiert (Rayner 2014).

Mit dem zunehmenden Einsatz von SaaS erhört sich einerseits der Bedarf für die Integration zwischen SaaS-Anwendungen untereinander. Beispielsweise nutzen in den USA einige Unternehmen parallel die beiden SaaS-Anwendungen Salesforce CRM auch NetSuite ERP. Andererseits - und dies dürfte das weitaus häufigere Szenario sein - besteht der Wunsch nach Integration zwischen SaaS- und bestehenden On-Premise-Anwendungen. Ein verbreitetes Szenario ist etwa der Abgleich von Kunden-, Produkt- und Auftragsdaten zwischen Salesforce, SAP ERP und dem Data Warehouse SAP BW.

Bisher wurden die Anwendungen zum einen über anwendungsspezifische Punktzu-Punkt-Integrationskomponenten miteinander verbunden (Sun et al. 2007). So existieren z. B. für Salesforce auf dem Salesforce AppExchange-Markplatz dedizierte Cloud-basierte Erweiterungen für die Anbindung an ein SAP ERP-System oder ein NetSuite ERP. Der Nachteil von Punkt-zu-Punkt-Verbindungen ist jedoch die hohe Schnittstellenanzahl, falls jede Anwendung mit jeder anderen kommunizieren muss. Zum anderen werden SaaS-Anwendungen auch mittels klassischer Enterprise Application Integration (EAI)-Plattformen integriert. Beispielsweise kann Salesforce mit Hilfe von SAP Process Integration an ein SAP ERP-System angebunden werden. EAI-Plattformen sind jedoch zum Teil sehr komplex und erfordern einen dedizierten Betrieb.

Eine Alternative zur direkten Anbindung zwischen Anwendungen und zu EAIPlattformen sind Cloud-basierte Integrationsplattformen. Sie versprechen die Vorteile von Cloud-Lösungen (z. B. geringe Kapitalbindung, hohe Flexibilität, wenig erforderliches IT-Know-how) in Kombination mit dem Nutzen von EAI-Plattformen (z. B. Reduktion der Schnittstellen-Anzahl durch zentralen Hub, standardisierte Anwendungsadapter). Das Schweizer Pharmaunternehmen Novartis ist ein promi- 
nentes Beispiel für die Verwendung der Cloud-basierten Integrationslösung Dell Boomi.

\section{Plattformen zur Anwendungsintegration}

\subsection{Vorausgehende Entwicklungen}

Ende der 1990er Jahre wurde der Begriff „Enterprise Application Integration“ erstmalig geprägt. Trotz der verstärkten Einführung von standardisierten ERP-Systemen sahen sich viele Unternehmen mit einer großen Heterogenität ihrer Anwendungslandschaft konfrontiert. Neben den ERP-Systemen existierten eigenentwickelte Mainframe-Anwendungen genauso wie abteilungsspezifische Client-Server-Systeme. Anforderungen wie die Verknüpfung von internen Geschäftsprozessen, die Vernetzung zwischen Unternehmen oder der elektronische Handel erforderten die Integration von Altanwendungen, ERP-Systemen und neuen Anwendungen wie E-Commerce-Systemen (Linthicum 2000, S. 7 ff.; Ruh et al. 2000, S. 1 ff.). Die direkte Punkt-zu-Punkt-Integration zwischen Systemen führte allerdings zu einer hohen Schnittstellenkomplexität und zu hohen Folgekosten.

Der EAI-Ansatz sieht die Integration auf folgenden Ebenen vor (Linthicum 2000, S. 21; Ruh et al. 2000, S. 19):

1. Daten (z. B. Synchronisation zweier Datenbanken)

2. Funktion: Anwendungsinterface, Service oder Methode (Funktionsaufruf zwischen Anwendungen)

3. Benutzeroberfläche (z. B. Single Sign-on oder Integration von Benutzeroberflächen über Portale)

4. Geschäftsprozess (z. B. Verknüpfung von Anwendungen und manuellen Arbeitsschritten über Workflows)

Die Kernidee von EAI ist die Verknüpfung der Anwendungen über eine zentrale, anwendungsunabhängige Middleware-Komponente (Ruh et al. 2000, S. 2 f.). Anstatt jede Anwendung direkt mit jeder anderen zu verbinden, werden lediglich Verbindungen von jeder Anwendung zur „EAI-Plattform“ geschaffen. Diese gewährleistet in der Regel den asynchronen Austausch von Daten mittels Nachrichten zwischen Anwendungen zur Integration auf Daten- und Funktionsebene. Typische EAI-Bestandteile waren nachrichtenorientierte Middleware, Transaktionsmonitore für die transaktional gesicherte Anwendungskommunikation und standardisierte Anwendungsadapter für die Verbindung der Anwendungen mit der Plattform (Ruh et al. 2000, S. 39 ff.). Mit der Zeit erweiterten die Anbieter die EAI-Plattformen jedoch um Webportal- und Business Process Management (BPM)-Komponenten zur Integration auf Ebene Benutzeroberfläche bzw. Geschäftsprozess.

Während bei EAI vor allem die Technik im Mittelpunkt steht, ist die Serviceorientierte Architektur (SOA) ein eher konzeptioneller Ansatz. Über die Anwendungslandschaft hinweg werden wiederverwendbare, geschäftsprozessnahe Dienste (,Services“) definiert, die über standardisierte Schnittstellen von anderen Diensten genutzt werden können (Krafzig et al. 2005, S. 56 ff.). Dabei werden oft offene Web- 
standards zur synchronen Kommunikation wie das Simple Object Access Protocol (SOAP) oder der Representational State Transfer (REST) zum Aufruf von Diensten genutzt.

Parallel zur Weiterentwicklung der Integrationsansätze entwickelte sich das Cloud-Computing. Im Gegensatz zum Eigenbetrieb von IT-Ressourcen (,On-Premise“) wird unter Cloud-Computing eine Dienstleistung verstanden, bei der virtuelle und selbstverwaltete Dienste, Anwendungen oder Ressourcen automatisch, flexibel und skalierbar über das Internet bereitgestellt werden. In der Regel werden sie verbrauchsorientiert abgerechnet und setzen keine langfristige Kapitalbindung oder Know-how voraus. SaaS ist eine spezifische Ausprägung des Cloud-Computing (Repschläger et al. 2010; Tietz et al. 2011).

\subsection{Cloud-basierte Integrationsplattformen}

Nachdem die ursprünglichen EAI-Plattformen klassische On-Premise-Software waren, entwickelten sich ab 2007 die ersten Integrationsplattformen in der Cloud. Diese werden nachfolgend als „Cloud-basierte Integrationsplattformen“ bezeichnet und wurden von Gartner wie folgt beschrieben:

[...] a suite of cloud services enabling development, execution and governance of integration flows connecting any combination of on premises and cloudbased processes, services, applications and data within individual, or across multiple, organizations. (Pezzini und Lheureux 2011)

Die Hersteller dieser Integrationsplattformen versprechen den einfachen grafischen Entwurf der Integrationslogik (nachfolgend „Integrationsprozess“), die Nutzung einer Vielzahl existierender Anwendungsadapter und vorhersehbare Kosten aufgrund nutzungsorientierter Verrechnungsmodelle ${ }^{1}$.

Die Plattformen sind mandantenfähige Systeme auf einer Cloud-Infrastruktur, in der sich verschiedene Kunden eine Instanz teilen. Die Entwicklung, Ausführung und Verwaltung der Integrationsprozesse erfolgt ganz oder teilweise in der Cloud. Im Unternehmen können sie sowohl für die Integration zwischen SaaS-Anwendungen als auch für die Anbindung von SaaS mit den On-Premise-Anwendungen genutzt werden.

Für die Anbindung der On-Premise-Anwendungen ${ }^{2}$ bieten die Hersteller in der Regel einen lokalen Software-Agenten für die Kommunikation zwischen lokalen Anwendungen und Cloud, der innerhalb der Unternehmens-Firewall installiert wird (Abb. 1, Variante A). Dadurch wird die On-Premise-Anbindung vereinfacht und die Kommunikation zwischen lokalen Anwendungen und Integrationsplattform verschlüsselt. Allerdings werden die Integrationsdaten an die Cloud-basierte Plattform und die beteiligen SaaS-Anwendungen übertragen. Als Alternative bieten einige Plattformen lokale Agenten als vollwertige lokale Laufzeitumgebung für die Integration an (Abb. 1, Variante B). In diesem Fall ist das Unternehmen selbst für die Integrationsinfrastruktur verantwortlich, allerdings werden die Integrationsdaten lediglich

\footnotetext{
${ }^{1}$ Vgl. z. B. www.boomi.com/integration (Zugriff am 5.5.2015)

${ }^{2}$ Dadurch entfällt z. B. die Notwendigkeit für eine VPN-Verbindung.
} 


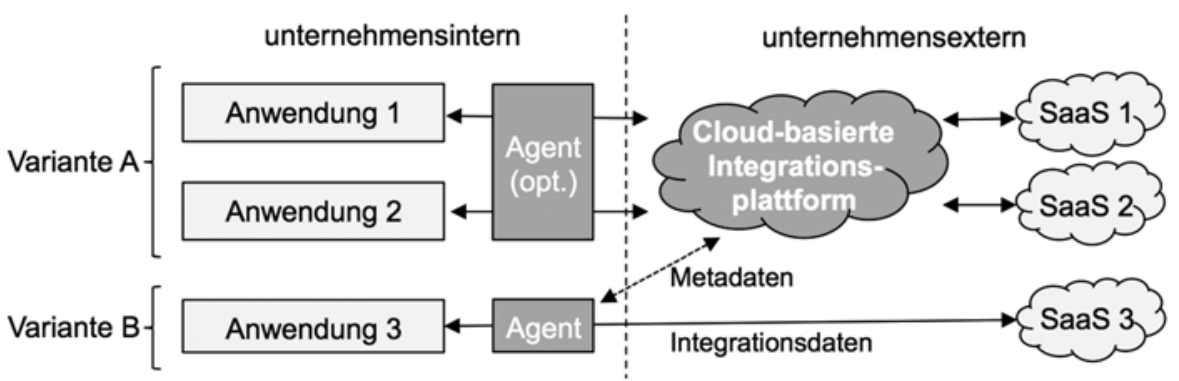

Abb. 1 Cloud-basierte Integrationsplattform im Anwendungskontext eines Unternehmens (eigene Darstellung)

zwischen On-Premise- und SaaS-Anwendungen ausgetauscht. Die Integrationsplattform dient lediglich zur Administration und tauscht mit dem lokalen Agenten ausschließlich Metadaten aus (z. B. Datenstrukturen).

Die Sicherheitsaspekte, die für Cloud-Angebote allgemein gelten, müssen auch für die Cloud-basierten Integrationsplattformen beachtet werden (vgl. z. B. Subashini und Kavitha 2011). Die Hersteller nutzen in der Regel eine ISO 27001 zertifizierte Infrastruktur (z. B. Amazon Web Services). Für den Webzugriff zur Administration und die Kommunikation mit On-Premise-Anwendungen werden unterschiedlich stark verschlüsselte HTTPS-Verbindungen verwendet. Die Kommunikation mit SaaS erfolgt - abhängig von den Möglichkeiten des jeweiligen SaaS - nach unterschiedlichen Standards wie HTTPS oder WS-Security. Falls keine lokale Laufzeitumgebung genutzt wird, werden die Integrationsdaten in der Cloud lediglich verarbeitet, jedoch nicht gespeichert. Angaben, ob die Metadaten auf den Plattformen verschlüsselt werden, machen die Anbieter nicht.

Der funktionale Fokus der Cloud-basierten Integrationsplattformen liegt auf der Integration auf Daten- und Funktionsebene. Die Integration auf Ebene der Geschäftsprozesse z. B. über BPM-Komponenten wird nur selten abgedeckt, die Integration auf Ebene der Benutzeroberflächen ist nicht Bestandteil der Plattformen. Die Funktionsbausteine der Cloud-basierten Plattformen unterscheiden sich jedoch nicht von denen klassischer EAI-Plattformen (vgl. Ring 2000):

- Prozessmanagement (z. B. Integrationsprozesse)

- Transformation (z. B. Mapping, Routing, Synchronisation)

- Konnektivität (z. B. Adapter, Kopplungsmechanismen)

- Administration (z. B. Monitoring)

- Entwicklung (z. B. Modellierung)

Die Prozessmanagement-Funktionalität dient der Ausführung der Transformationsoperatoren. In der Regel werden die Transformationsoperatoren mit Logikoperatoren (z. B. Verzweigungen) zu mehrstufigen Integrationsprozessen verknüpft. Sie können Daten sowohl im Batch-Modus als auch einzeln verarbeiten und zeitlich geplant oder ereignisorientiert von anderen Anwendungen gestartet werden. Zum Teil unterstützen die Integrationsplattformen Standards wie die Business Process Execution Language (BPEL) oder die Business Process Modelling Notation (BPMN) für die Gestaltung 
der Integrationsprozesse. Vielfach existieren Vorlagen für die Integrationsprozesse („Integrationstemplates“), um den Entwicklungsprozess zu beschleunigen.

Die Integrationsplattformen bieten umfangreiche Funktionen zur Transformation der Daten zwischen verschiedenen Datenquellen. Verschiedene Operatoren erlauben z. B. die Abbildung zwischen unterschiedlichen Datenschemata und Datenformaten. Hierzu werden Abbildungsregeln zwischen den verschiedenen Datenmodellen definiert (z. B. Abbildung von „Vorname Nachname“ auf „Name“). Weitere Beispiele für Operatoren sind Filter zur Selektion von Daten oder zur Bereinigung von fehlerhaften Datenquellen. Vielfach bieten die Integrationsplattformen Standardvorlagen z. B. für die Datenfilterung oder Abbildung zwischen Datenformaten.

Vorgefertigte Adapter ermöglichen die Anbindung von Cloud- oder On-PremiseAnwendungen und Datenquellen. Sie reichen von einfachen Datei- oder HTTP-Adaptern über Adapter für Geschäftsanwendungen (z. B. SOAP-Adapter für Salesforce. com, Bapi/Idoc für SAP) bis hin zu Adaptern für den zwischenbetrieblichen Datenaustausch (z. B. EDI, ebXML). Häufig existieren Online-Marktplätze, über die Adapter der Hersteller oder Dritter bezogen werden können. Die Integrationsplattformen erlauben außerdem Integrationsprozesse als eigenständige Dienste bereitzustellen, die dann wiederum von anderen Diensten aufgerufen werden können (z. B. innerhalb einer SOA).

Die Kopplungsmechanismen der Plattformen entsprechen denen aktueller OnPremise-Middleware-Produkte und erlauben die Verknüpfung der Anwendungen über Dateitransfers, asynchrone Nachrichten und synchrone Dienstaufrufe. Ebenso unterstützen manche Plattformen die Ausführung von Transaktionen, die z. B. im Fehlerfall einer Anwendung das „Rollback“ in anderen Anwendungen ermöglichen.

Administrationsfunktionen der Plattformen sind z. B. das Deployment und Monitoring der Integrationsprozesse und die Konfiguration der Laufzeitumgebung (z. B. Auswahl der Rechenleistung und geografischen Verortung). Schließlich verfügen alle Plattformen über Entwicklungswerkzeuge. Sie erlauben die grafische Modellierung, die Schritt-für-Schritt-Erstellung und die Konfiguration von Integrationsprozessen. Die Plattformen ermöglichen ferner die Eigenentwicklung von Adaptern und Transformationsoperatoren und bieten dafür SoftwareentwicklungsKits (SDK).

\section{Aktuelle Integrationsplattformen}

Mittlerweile existiert eine Vielzahl von Cloud-basierten Integrationsplattformen sowohl von etablierten Herstellern wie IBM, Microsoft und SAP als auch von neuen Anbietern wie MuleSoft, Jitterbit und Dell (Pezzini et al. 2015). Nachfolgend werden die in Tab. 1 dargestellten Produkte, die von Pezzini et al. 2015 im ,,magischen Quadranten" besonders positiv bewertet wurden, detaillierter vorgestellt.

\subsection{Dell Boomi Atomsphere}

Die Firma Boomi wurde im Jahr 2000 gegründet und veröffentlichte 2007 die Integrationsplattform „Atomsphere“. Im Jahr 2010 erfolgte die Übernahme von Boomi 
Tab. 1 Überblick über die betrachteten Integrationsplattformen (eigene Darstellung)

\begin{tabular}{|c|c|c|c|c|}
\hline Funktionalität & $\begin{array}{l}\text { Dell Boomi } \\
\text { Atomsphere }\end{array}$ & $\begin{array}{l}\text { MuleSoft } \\
\text { Cloudhub }\end{array}$ & $\begin{array}{l}\text { Informatica } \\
\text { Cloud }\end{array}$ & SAP HCI \\
\hline \multicolumn{5}{|l|}{ Prozessmgmt } \\
\hline Prozessarten & $\begin{array}{l}\text { Mehrstufige Pro- } \\
\text { zesse (Batch/Ein- } \\
\text { zel, Plan/Ereignis, } \\
\text { eigene Syntax) }\end{array}$ & $\begin{array}{l}\text { Mehrstufige Pro- } \\
\text { zesse (Batch/Ein- } \\
\text { zel, Plan/Ereignis, } \\
\text { eigene Syntax) }\end{array}$ & $\begin{array}{l}\text { 1. Batch (geplant) } \\
\text { 2. mehrst. Prozes- } \\
\text { se (Batch/Einzel, } \\
\text { Plan/Ereignis) } \\
\text { 3. Workflows } \\
\text { (ActiveVOS, z. B. } \\
\text { BPEL) }\end{array}$ & $\begin{array}{l}\text { 1. Batch (Plan/ } \\
\text { Ereignis) } \\
\text { 2. mehrst. Prozesse } \\
\text { (Batch/Einzel, } \\
\text { Plan/Ereignis, } \\
\text { BPMN) }\end{array}$ \\
\hline Prozessausführung & Lokal/Cloud & Cloud & Lokal & Cloud \\
\hline Transformation & $>20$ Operatoren & $\begin{array}{l}>110 \text { Operatoren } \\
\text { (eigene können } \\
\text { entwickelt werden) }\end{array}$ & $\begin{array}{l}>40 \text { Operatoren } \\
\text { (PowerCenter) }\end{array}$ & $\begin{array}{l}>30 \text { Operato- } \\
\text { ren (Integration } \\
\text { Designer) }\end{array}$ \\
\hline \multicolumn{5}{|l|}{ Konnektivität } \\
\hline $\begin{array}{l}\text { On-Premise } \\
\text { Anbindung }\end{array}$ & $\begin{array}{l}\text { Software-Agent } \\
\text { (Win./Linux) }\end{array}$ & direkt & $\begin{array}{l}\text { Software-Agent } \\
\text { (Win./Linux) }\end{array}$ & $\begin{array}{l}\text { Software-Agent } \\
\text { (Win./Linux) }\end{array}$ \\
\hline Adapter & $\begin{array}{l}>85 \text { (z. B. SAP } \\
\text { ERP, Oracle } \\
\text { E-Business, } \\
\text { Salesforce) }\end{array}$ & $\begin{array}{l}>117 \text { (z. B. } \\
\text { SAP Business, } \\
\text { Salesforce, Oracle } \\
\text { E-Business) }\end{array}$ & $\begin{array}{l}>121 \text { (z. B. Net- } \\
\text { Suite, SAP ERP, } \\
\text { Salesforce, Micro- } \\
\text { soft Dynamics) }\end{array}$ & $\begin{array}{l}\text { Zahlreiche SAP- } \\
\text { Adapter (z. B. } \\
\text { HANA, ERP, } \\
\text { SuccessFactors) }\end{array}$ \\
\hline Kopplung & $\begin{array}{l}\text { Datei, Nachricht, } \\
\text { Funktionsaufruf }\end{array}$ & $\begin{array}{l}\text { Datei, Nachricht, } \\
\text { Funktionsaufruf }\end{array}$ & $\begin{array}{l}\text { Datei, Nachricht, } \\
\text { Funktionsaufruf }\end{array}$ & $\begin{array}{l}\text { Datei, Nachricht, } \\
\text { Funktionsaufruf }\end{array}$ \\
\hline Administration & Via Web & Via Web & Via Web & $\begin{array}{l}\text { Via Web/Integra- } \\
\text { tion Designer }\end{array}$ \\
\hline Entwicklung & Via Web & $\begin{array}{l}\text { Anypoint Studio } \\
\text { (Win./Linux/Mac) }\end{array}$ & $\begin{array}{l}\text { Via Web (Daten)/ } \\
\text { PowerCenter } \\
\text { (komplexe Prozes- } \\
\text { se, Win./Linux) }\end{array}$ & $\begin{array}{l}\text { Via Web (Daten)/ } \\
\text { Integration De- } \\
\text { signer (komplexe } \\
\text { Prozesse, Win./ } \\
\text { Linux/Mac) }\end{array}$ \\
\hline \multicolumn{5}{|l|}{ Sicherheit } \\
\hline $\begin{array}{l}\text { Kommunikation } \\
\text { mit Plattform }\end{array}$ & $\begin{array}{l}\text { HTTPS/RC4 } 128 \\
\text { bit }\end{array}$ & HTTPS/k. A. & $\begin{array}{l}\text { HTTPS/Verisign } \\
128 \text { bit v3 }\end{array}$ & HTTPS/256 bit \\
\hline $\begin{array}{l}\text { Verschlüsselung } \\
\text { auf Plattform }\end{array}$ & k. A. & $\begin{array}{l}\text { Warteschlagen } \\
\text { verschlüsselbar }\end{array}$ & k. A. & k. A. \\
\hline
\end{tabular}

durch Dell. Mit der Atomsphere-Plattform können Integrationsprozesse vollständig Cloud-basiert entwickelt, ausgeführt und verwaltet werden.

Die grafische Modellierung der Batch- oder ereignisbasierten-Integrationsprozesse findet ausschließlich in einer Weboberfläche statt (Abb. 2). Ausgangspunkt eines Prozesses sind prozessierbare Dokumente. Ein Dokument kann ein „Flatfile“, ein Datenbankeintrag, eine XML- oder eine EDI-Datei sein. Dokumente werden von Adaptern (,Connectors“) erzeugt, die über verschiedene Operatoren verfügen (z. B. „Get customers“ oder „Update customers“). Es existiert eine große Anzahl vorhandener Adapter, die bei Änderungen der Schnittstellen aktualisiert werden. Abbildungsoperatoren (,Profile“) erlauben die Transformation von Dokumenten zwischen Datenschemata. Die Datenschemata können manuell erstellt oder für manche Datenquellen automatisch generiert werden. Beispielsweise kann der Salesforce-Adapter das Schema eines Salesforce-Kundenobjekts erzeugen, das dann auf ein Ziel (z. B. CSV) abgebildet wird. Die Funktion „Boomi-Suggest“ ist hervorzuheben, die auf 


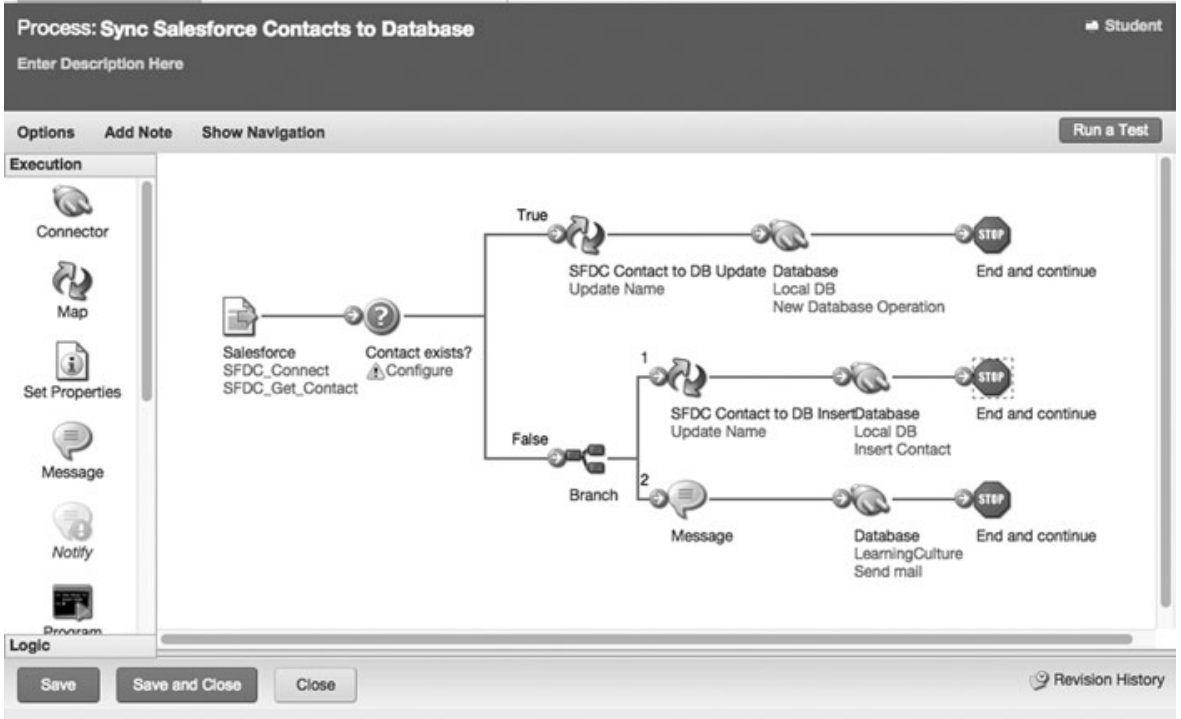

Abb. 2 Musterprozess der Atomsphere-Plattform. (Quelle: https://ondemand.boomi.com. Zugegriffen am 3. Mai 2015)

Basis der Abbildungen anderer Atomsphere-Nutzer automatisch Vorschläge eigene Abbildungen generiert. Das Deployment der Integrationsprozesse erfolgt in der skalierbaren „Atom“-Laufzeitumgebung, die auch lokal installiert werden kann.

\subsection{MuleSoft Cloudhub}

Cloudhub wurde 2012 von der Firma Mulesoft vorgestellt und basiert ursprünglich auf dem Open Source Projekt „Mule Enterprise Service Bus“ (Mule ESB). Aufgrund der Open Source-Vergangenheit verfügt MuleSoft über eine breite Kunden- und Entwicklerbasis.

Die Modellierung der „Mule Flows“-Integrationsprozesse in der Eclipse-Anwendung Anypoint Studio ist vergleichbar mit dem Entwurf in Boomi Atomsphere. Innerhalb eines Projekts können ein oder mehrere Mule Flows definiert werden, in denen „Message Processors“ grafisch verknüpft werden. Die komplexen Batch- oder eventbasierten Prozesse können alternativ als XML-Dokument direkt editiert werden. Eine Besonderheit ist die große Flexibilität der Datenmanipulation: in Operatoren können neben der „Mule Expression Language“ auch Java, Groovy, Python, Ruby oder PHP genutzt werden. Über einen Marktplatz und die Open Source Community werden diverse Prozessvorlagen - zum Teil kostenlos - bereitgestellt.

Ein Projekt wird während der Testphase zunächst auf einem lokalen Mule ESB ausgeführt und danach in der Cloud-Umgebung „Cloudhub“ installiert, die auf der Amazon Web Services-Infrastruktur (AWS) basiert. Während des Deployments kann - entsprechend zu den AWS Regionen - der geografische Ort der Laufzeitumgebung gewählt werden (z. B. EU). 


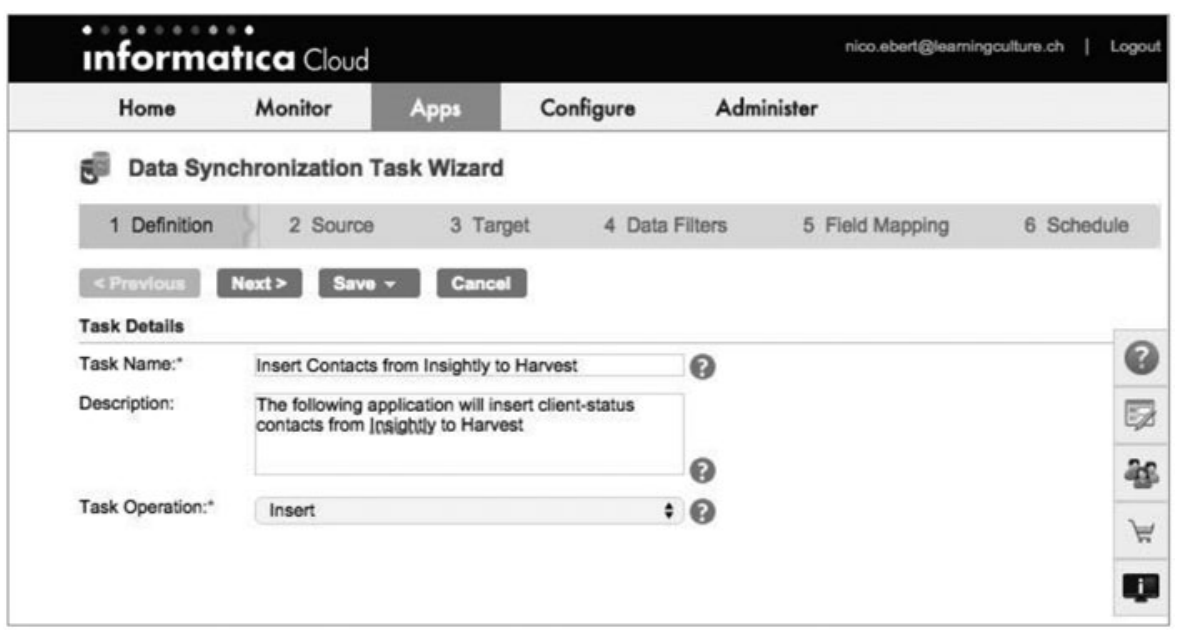

Abb. 3 Batch-Synchronisation mit der Informatica Cloud. (Quelle: https://app.informaticaondemand. com/ma/. Zugegriffen am 29. April 2015)

\subsection{Informatica Cloud}

Das auf die Datenintegration spezialisierte Unternehmen Informatica stellte im Jahr 2012 die Informatica Cloud vor. Sie verfügt über separate Anwendungen zur BatchDatenintegration und zur Ausführung von komplexeren Integrationsprozessen. Letztere basiert zum Teil auf dem Business Process Management- Produkt ActiveVOS des 2013 übernommenen Anbieters active endpoints.

Für die Batch-Datenintegration existieren Wizard-basierte Webanwendungen zur Beurteilung von Datenquellen („Data Assessment“), zur Replikation von Anwendungsdaten auf Dateien oder Datenbanken (,Data Replication“) und zur Synchronisation von Daten zwischen Anwendungen („Data Synchronisation“) (Abb. 3).

Komplexere Integrationsprozesse können mit der Anwendung PowerCenter modelliert und anschließend auf der Informatica Cloud deployed werden. Alternativ kann eine webbasierte grafische Modellierungsumgebung genutzt werden, die auf ActiveVOS basiert. Sie erlaubt es, zustandsbehaftete Abläufe z. B. in BPEL oder BPMN zu modellieren. Analog zu den anderen Plattformen existieren für die Informatica Cloud eine Vielzahl von Adaptern, Verarbeitungsoperatoren und vordefinierten Prozessvorlagen. Eigene Adapter werden mit dem Cloud Connector Toolkit entwickelt.

Anders als bei den anderen Plattformen ist die Laufzeitumgebung (,Secure Agent") für die Integrationsprozesse standardmäßig lokal ${ }^{3}$ - selbst wenn zwei SaaSAnwendungen integriert werden sollen.

\subsection{SAP HANA Cloud Integration}

Im Jahr 2012 wurde von SAP die „Netweaver Cloud Integration“ angekündigt, die seit 2013 - in Anlehnung an die Cloud-Plattform HANA (vgl. Prassol 2015) - als

\footnotetext{
${ }^{3}$ Denkbar ist die Installation auf einer Cloud-Infrastruktur wie Amazon Web Services.
} 


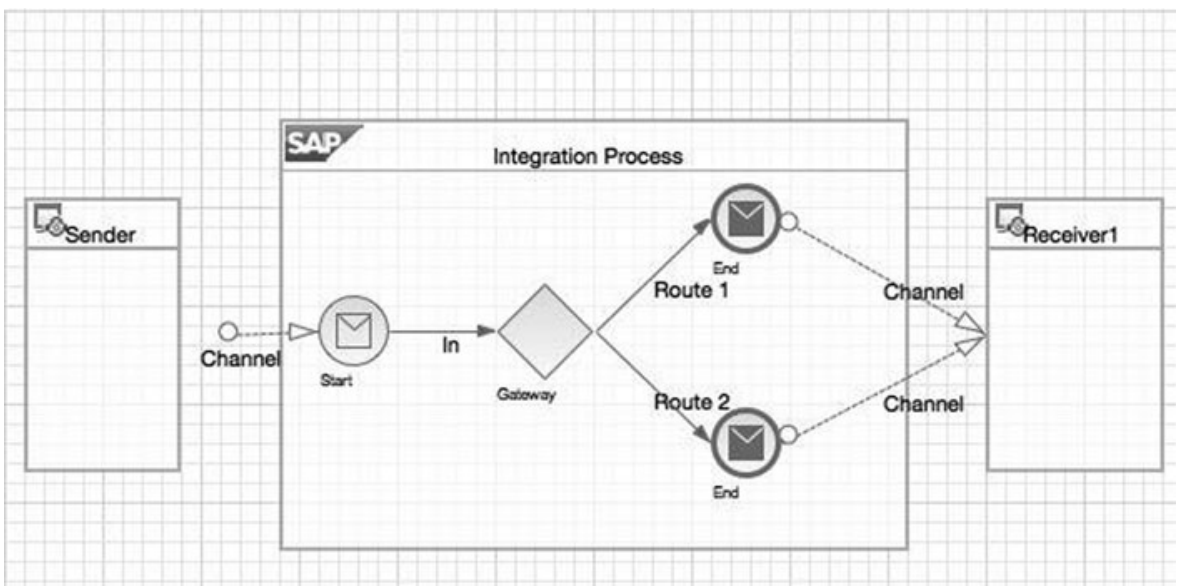

Abb. 4 Musterprozess im SAP Integration Designer (Version 2.3.0.)

„HANA Cloud Integration“ (HCI) bezeichnet wird und aus zwei separaten Anwendungen besteht.

HCI for Data Services wird von SAP primär als webbasiertes ETL-Tool für den Batch-Austausch von Daten aus On-Premise und SaaS-Systemen in die Hana Cloud betrachtet. „Datastores“ beinhalten sowohl Verbindungsinformationen zu einer Datenquelle als auch Schema-Informationen. „Data Flows“ beschreiben die Transformation zwischen Datastores und können geplant oder ereignisbasiert aufgerufen werden. Sie können als grafische Abläufe mit Verarbeitungsoperatoren modelliert werden (z. B. zur Bereinigung der Daten). Ein Software-Agent ist für den Datentransfer in die HANA Cloud zuständig, wo die Flows ausgeführt werden.

Im Gegensatz zu HCI for Data Services erlaubt HCI for Process Integration die Modellierung und Ausführung von komplexen, ereignisbasierten Integrationsprozessen. Die Prozesse werden in einer Eclipse-basierten Umgebung in einer vereinfachten BPMNNotation modelliert (Abb. 4). Bestehende Inhalte (z. B. Daten-Mappings) aus der OnPremise-Anwendung SAP PI können importiert werden. SAP bietet eine große Anzahl von (teilweise komplexen) Adaptern und Prozessvorlagen für eigene On-Premise- und Cloud-Software. Der „SAP HANA Cloud Connector“ übernimmt die Verbindung zu OnPremise-Systemen. Die Integrationsprozesse finden innerhalb der HANA Cloud statt.

\section{Anwendungsbeispiel einer Cloud-basierten Integrationsplattform}

\subsection{Ausgangssituation und Herausforderungen}

Die LearningCulture GmbH ist ein 2013 gegründeter Schweizer Nachhilfeanbieter. Die Lehrkräfte besuchen die Schüler in der Regel wöchentlich zu Hause und unterstützen in den typischen Schulfächern. Ein administratives Team leistet die Kundenbetreuung, die Lehrerdisposition, das Rechnungswesen sowie die Lehrerbetreuung und -rekrutierung. 


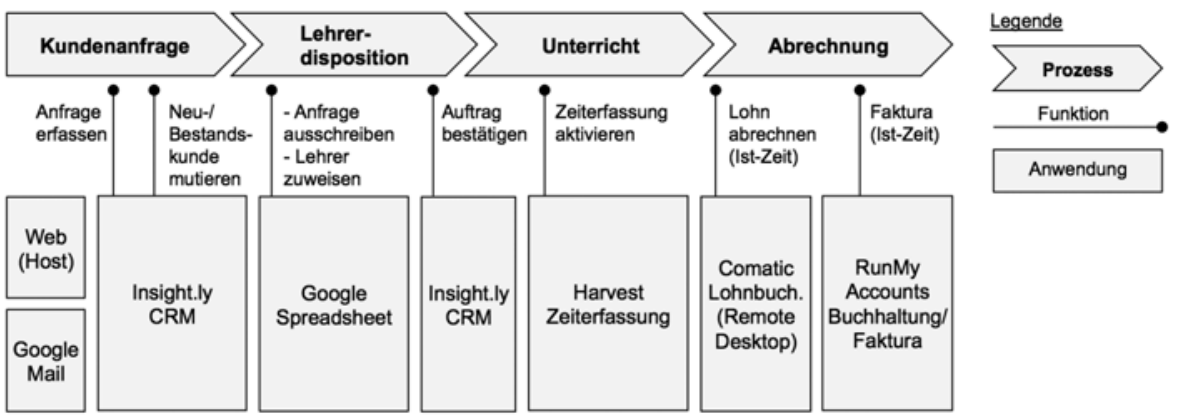

Abb. 5 Geschäftsprozess „Auftragsabwicklung“ mit unterstützenden IT-Anwendungen (eigene Darstellung)

Bereits mit Gründung des Unternehmens stand fest, dass sämtliche IT-Unterstützung aufgrund eines niedrigen IT-Budgets SaaS-basiert sein sollte. Die Auswahl der SaaS-Lösungen erfolgte nach dem Best-of-Breed-Ansatz, was vor allem im Fehlen eines geeigneten SaaS-ERP-Angebots begründet lag. Einen exemplarischen Geschäftsprozess mit dessen Anwendungsunterstützung zeigt Abb. 5.

Die Nachhilfeanfrage eines Kunden wird über verschiedene Kanäle entgegen genommen und im CRM-System mit den Kundendaten erfasst. Im Anschluss erfolgen mittels Google Spreadsheet die Benachrichtigung der Lehrer, die Abklärung der Verfügbarkeit eines Lehrers und dessen finale Zuweisung zur Anfrage. Nachfolgend findet die finale Auftragsbestätigung durch den Kunden und die Aktivierung der Zeiterfassung für den Lehrer statt. Die vom Lehrer auf Ebene Kunde und Auftrag erfassten Zeiten finden am Monatsende schließlich Eingang in die Anwendungen für die Lohnbuchhaltung und Buchhaltung/Faktura. Zwischen den insgesamt neun, nicht verbundenen IT-Anwendungen der LearningCulture GmbH liegt eine erhebliche Datenredundanz vor: z. B. müssen die Datenobjekt „Kunde“ und „Auftrag“ in mehreren Anwendungen erfasst und geändert werden. Die Mehrfacherfassung führt sowohl zu einem hohen zeitlichem Zusatzaufwand bei den zuständigen Sachbearbeitern als auch zu häufigen Erfassungsfehlern und Inkonsistenzen.

\subsection{Machbarkeitsstudie einer Cloud-basierten Integrationsplattform}

Da aufgrund fehlender Adapter keine direkte Kopplung der Anwendungen möglich war und keine klassische EAI-Plattform betrieben werden sollte, bestand das Ziel der Machbarkeitsstudie in der technischen Abklärung einer Integration mittels einer Cloud-basierten Integrationsplattform (Abb. 6). Sämtliche Anwendungen bieten zu diesem Zweck REST-Schnittstellen. Einzige Ausnahme bildet die gehostete Lohnbuchhaltungs-Anwendung, mit der lediglich Dateien via Netzlaufwerk ausgetauscht werden können. Als Plattform wurde für die Machbarkeitsstudie die Lösung von MuleSoft ausgewählt. Die Lösung SAP HCI wurde als zu SAP-spezifisch (z. B. zwingende Nutzung der HANA Plattform) und zu komplex beurteilt. Die Informatica Lösung setzte eine lokale Laufzeitumgebung voraus, was der SaaS-Strategie im Unternehmen widersprach. Die allgemein verfügbare Wissensbasis von Dell Boomi war schließlich zu gering, so dass zwangsläufig externe Unterstützung not- 


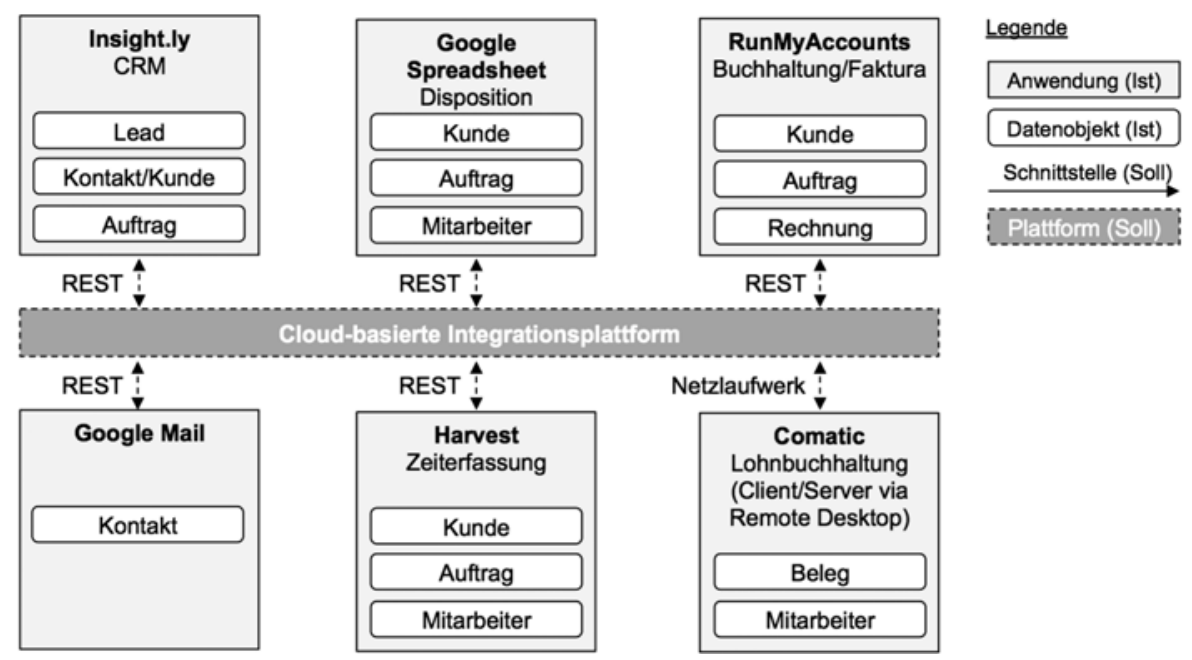

Abb. 6 Anwendungslandschaft mit geplanter Integrationsplattform (eigene Darstellung)

wendig gewesen wäre. MuleSoft überzeugte hingegen durch eine einfache Handhabung, eine breite Wissensbasis sowie eine Vielzahl von Adaptern auch für kleinere Unternehmensanwendungen.

In der Machbarkeitsstudie lag der Fokus auf der Unterstützung des Geschäftsprozesses aus Abb. 5. Zu diesem Zweck wurden insgesamt sieben Integrationsprozesse ausgewählt, z. B. zur Synchronisation von Kunden und Aufträgen von der CRM- zur Zeiterfassungsanwendung (vgl. Abb. 6). Da keine der involvierten Anwendungen Funktionen anderer Anwendungen aufrufen kann, wurden im Anypoint Studio planmäßig wiederkehrende Integrationsprozesse definiert, die ihrerseits die Funktionen aufrufen.

Nachfolgend wird die Synchronisation zwischen CRM- und Zeiterfassung detaillierter betrachtet. Wann immer ein Kunde und Auftrag im CRM-System angelegt wird, soll die Anlage ebenfalls im Zeiterfassungssystem erfolgen, da geleistete Zeiten von Lehrern auf Kundenaufträgen erfasst werden müssen. Die Synchronisation zwischen CRM- und Zeiterfassungssystem erfolgt einmal täglich, was aufgrund der mehrtätigen Auftragsabwicklung ausreichend ist. In Abb. 7 ist beispielhaft die vereinfachte Synchronisation des Kundenobjekts von der CRM- zur Zeiterfassungsanwendung dargestellt. Dabei handelt es sich um einen Batchprozess, der eine Eingabenachricht erzeugt (,Input“), je Datensatz eine Verarbeitung durchführt („Process Records“) und ein Abschlussevent erzeugt („On Complete“). Einmal täglich erfolgt die Abfrage aller Kontakte im CRM im JSON-Format per HTTP-GetMethode. Der Operator „JSON to MAP“ filtert dann zunächst diejenigen Kontakte, die als Kunde klassifiziert wurden. Im gleichen Schritt werden deren JSON-Daten zu einer Liste aus Schlüssel-Wert-Paaren (z. B. Schlüssel „Name“: Wert „Maier“) transformiert, die der anschließende Batchprozess Kunde für Kunde iteriert. Im Prozess „Send_Record_to_Harvest“" wird jedes Schlüssel-Wert-Paar einzeln in das XMLSchema der Zeiterfassungsanwendung konvertiert („MAP to XML“) und per HTTP- 


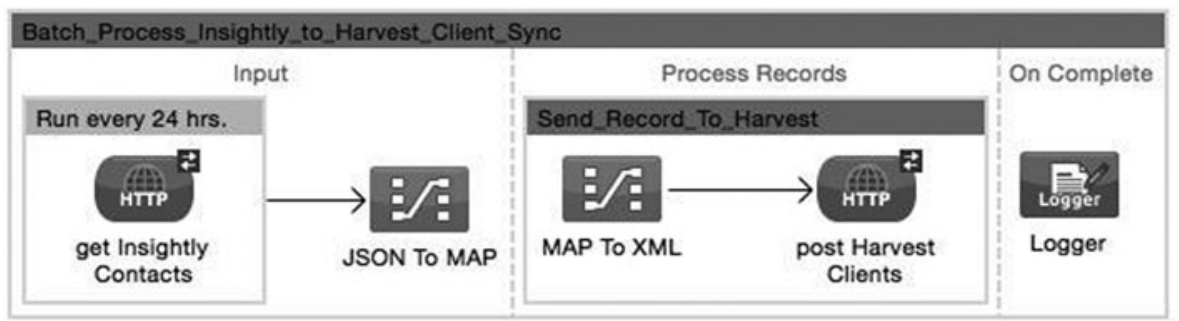

Abb. 7 Vereinfachter Integrationsprozess für Kundenobjekte von CRM zur Zeiterfassung (MuleSoft Anypoint Studio, Version 5.1.1.)

Post-Methode übergeben. Bereits vorhandene Kunden werden anhand des Namens ${ }^{4}$ identifiziert und ignoriert. Nachdem alle Kundendatensätze abgeglichen wurden, erfolgt im letzten Schritt die Erstellung eines Logfiles („Logger“).

Jeder Prozess wurde nach der Modellierung in der lokalen Laufzeitumgebung Mule ESB getestet. Anschließend erfolgte aus dem Anypoint Studio heraus das Produktivdeployment auf der Cloudhub-Plattform. In der Machbarkeitsstudie erwies sich ein Arbeiterprozess mit 0.1 virtuellen Prozessorkernen mit 500 MB RAM als ausreichend performant.

Insgesamt erwies sich die Integration der Anwendungen als problemlos. Der Prozessentwurf erforderte jedoch einerseits die umfangreiche Einarbeitung in die Anypoint-Umgebung. Andererseits war eine Auseinandersetzung mit der jeweiligen REST-API erforderlich, da keine Standard-REST-Adapter existierten und lediglich der generische HTTP-Adapter für die REST-Aufrufe genutzt werden konnte. Beides war aufgrund zahlreicher öffentlicher Beispiele durch Konfiguration vorhandener Operatoren machbar. Die laufende Wartung der Plattform beschränkt sich auf die Anpassung der Prozesse an geänderte Schnittstellen und die Skalierung der Laufzeitumgebung für geänderte Transaktionsvolumina.

\section{Fazit und Ausblick}

Aus dem Vergleich der Angebote und dem vorgestellten Fallbeispiel wurde ersichtlich, dass die Cloud-basierten Integrationsplattformen Alternativen zu bestehenden Integrationswerkzeugen sein können. Die dargestellten Angebote zeichnen sich durch eine einfache Bedienung und eine Vielzahl vorhandener Integrationsvorlagen und Anwendungsadapter aus. Die Adapter unterstützen unterschiedliche Sicherheitsstandards für die Kommunikation und können mittels SDKs auch selbst entwickelt werden. Neben dem Zugriff auf bestehende Services können einige der Plattformen Integrationsprozesse auch als eigene Dienste bereitstellen. Damit wird zusätzlich die Anbindung in eine bestehende SOA ermöglicht.

Häufig bieten die Plattformen lokale Software-Agenten, die in separierten Netzwerkbereichen installiert werden, den Zugriff auf On-Premise-Systeme vereinfachen

\footnotetext{
${ }^{4}$ Gemeinsamer Primärschüssel ist die automatisch generierte Kontakt-ID im CRM-System, die im Namens-Attribut des Kundenobjekts der Zeiterfassung hinterlegt wird.
} 
und den Datenaustauch zur Integrationsplattform verschlüsseln. Bei einigen Anbietern übernimmt der Agent auch die Funktion einer lokalen Laufzeitumgebung für die Integrationsprozesse, so dass lediglich Metadaten mit der Cloud ausgetauscht werden. Damit schwindet zwar der Vorteil der schnellen Skalierbarkeit, allerdings steigen die Informationssicherheit und die Kontrolle über die Daten, weil lediglich Metadaten in der Cloud verarbeitet werden. Unabhängig von der Art der Laufzeitumgebung werden auf den Plattformen nur die Metadaten gespeichert.

Je nach Integrationsszenario eignen sich die verschiedenen Plattformen allerdings unterschiedlich gut. Während ein lokaler Software-Agent im SaaS-zu-On-PremiseSzenario hilfreich ist, erweist sich dessen zwingende Vorgabe durch den Hersteller als Hindernis in einem reinen SaaS-zu-SaaS-Szenario. Ebenso wird deutlich, dass auch die Anwendungslandschaft maßgeblichen Einfluss auf die Zweckmäßigkeit einer Plattform hat. So eignet sich die SAP HCI Plattform mit ihrer engen Kopplung an SAP HANA und vielen SAP-spezifischen Adaptern vor allem in einer SAP-dominierten Landschaft. In einer Best-of-Breed-Anwendungslandschaft hingegen eignen sich eher Plattformen, die über eine große Anzahl von Adaptern für unterschiedliche Hersteller verfügen.

Obwohl die Cloud-basierten Integrationsplattformen schon einige Jahre existieren, werden sie scheinbar vor allem im US-amerikanischen Markt genutzt ${ }^{5}$. Die Akzeptanz im europäischen oder deutschsprachigen Raum ist vermutlich eher gering, wie die Suche im Web nach Fallbeispielen nahe legt. Einige Hersteller wollen dies jedoch durch die stärkere Etablierung von lokalen Partnernetzwerken für die Beratung und Systemeinführung ändern.

\section{Literatur}

Krafzig D, Banke K, Slama D (2005) Enterprise SOA: service-oriented architecture best practices. Prentice Hall Professional, New Jersey

Linthicum DS (2000) Enterprise application integration. Addison-Wesley, Boston

Pezzini M, Lheureux B (2011) Integration platform as a service: moving integration to the cloud. Gartner, Stamford, S 1-9

Pezzini M, Natis YV, Malinverno P, Iijima K, Thompson J, Thoo E, Guttridge K (2015) Magic quadrant for enterprise integration platform as a service. Gartner, Stamford, S 1-35

Prassol P (2015) SAP HANA als Anwendungsplattform für Real-Time Business. HMD Prax Wirtschinform 52:358-372

Rayner N (2014) Survey Analysis: Adoption of Cloud ERP, 2013 Through 2023. Gartner, Stamford

Repschläger J, Pannicke D, Zarnekow R (2010) Cloud Computing: Definitionen, Geschäftsmodelle und Entwicklungspotenziale. HMD Prax Wirtschinform 47:6-15

Ring K (2000) EAI: Making the right connections. Ovum Reports, Boston

Ruh WA, Maginnis FX, Brown WJ (2000) Enterprise application integration: A Wiley tech brief. John Wiley \& Sons, New York

Subashini S, Kavitha V (2011) A survey on security issues in service delivery models of cloud computing. J Netw Comput Appl 34:1-11

Sun W, Zhang K, Chen S-K, Zhang X, Liang H (2007) Software as a service: an integration perspective. In: Krämer B, Lin K-J, Narasimhan P (Hrsg) Service-oriented computing - ICSOC 2007. Springer, Berlin, S 558-569

Tietz V, Blichmann G, Hübsch G (2011) Cloud-Entwicklungsmethoden. Inform-Spektrum 34:345-354

${ }^{5}$ Vgl. www.boomi.com/customers oder www.mulesoft.com/case-studies. 